\title{
IORT: clinical indications
}

\author{
M. Krengli, L. Masini, D. Beldi
}

\section{Introduction}

The concept of using ionizing radiation to treat the tumour bed or the tumour itself during a surgical procedure was envisaged for the first time beginning of 1900 and the very first treatments were described by Beck ${ }^{4}$ and Finsterer ${ }^{15}$ for advanced gastro-intestinal tumours. In the 1930s, the treatment of six patients with advanced gastric cancer was reported by Eloesser at Stanford ${ }^{14}$. The technological progress allowed to substantially improve this treatment modality in the early 1960s when cobalt-60 units first and electron beam accelerators then became available. Clinical trials started in Japan at the Kyoto University and in US at Massachusetts General Hospital (MGH) and Mayo Clinic. For many years, the treatments were performed by using conventional machines moving the patients from the operating room to the treatment room inside the radiotherapy department. Only in a few institutions like Howard University, MGH, and Mayo Clinic dedicated linear accelerators were installed in the operating rooms to facilitate the procedure. In Europe, a number of institutions in various countries started IORT clinical activity in the 1980s using electron beam and in some cases orthovoltage irradiation. The pioneering centres were Caen (France) in 1983, Pamplona (Spain), Innsbruck (Austria) in 1984, Lyon (France) and Milan (Italy) in 1985. The first tumours treated included mostly the gastro-intestinal and genito-urinary tracts and the retroperitoneal sarcoma. The implementation of dedicated machines able to deliver electron beams of 9-12 MeV in the late 1990s and the foundation of the International Society of Intra-Operative Radiation Therapy (ISIORT) in 1998 lead to a new era of IORT. At present, about 86 centres in at least 16 countries are clinically active worldwide and the number will probably increase consistently in the near future.

Division of Radiotherapy

University of Piemonte Orientale "Amedeo Avogadro"

Hospital "Maggiore della Carità"

Novara (Italy)

\section{Clinical indications}

Historically, the most treated tumour locations are represented by colorectal carcinoma, pancreatic carcinoma, gastric carcinoma, biliary carcinoma, soft tissue sarcoma, gynecologic malignancies, and bladder cancer but in the last years new indications emerged like breast cancer, head and neck tumours, prostate carcinoma, intra-thoracic malignancies, and brain tumours.

\section{Colorectal cancer}

IORT has been used for advanced primary or recurrent colorectal cancer.

The Mayo Clinic experience shows an improvement of 3year local control from $24 \%$ to $85 \%$ and 3 -year survival from $24 \%$ to $55 \%$ for the patients with locally advanced tumours treated with external radiotherapy + IORT in comparison with those treated with external irradiation only ${ }^{23,24}$. The accomplishment of gross total resection rather than subtotal tumour removal seemed to be a factor able to increase the rate of local control and DFS as demonstrated by the study performed at MGH in 64 patients affected by locally advanced primary lesions ${ }^{62}$. A number of European studies combined IORT with doses of 10-17 Gy and preoperative fractionated external beam radiotherapy with doses of 38-54 Gy obtaining quite favourable results: pelvic recurrence occurred in 3$13 \%$ of cases and 5 -year survival ranged from $60 \%$ to $82 \%$, $11,13,27,34,57$.

The long-term survival of the patients affected by recurrent rectal cancer is very poor accounting approximately less than $5 \%$ with standard techniques. At MGH, 41 patients treated with IORT obtained 5-year local control and DFS of $21 \%$ and $47 \%$ with negative margins and $7 \%$ and $21 \%$ with positive margins respectively ${ }^{61}$. The results of IORT in addiction to surgical resection were compared with those of surgery alone in locally recurrent rectal cancer at Mayo Clinic: 3- and 5-year survival were $43 \%$ and $19 \%$ with IORT and $18 \%$ and $7 \%$ without IORT ${ }^{62}$. The most relevant prognostic factor resulted the extent of residual disease after surgery. The MGH experience of a treatment program of high-dose preoperative irradiation, surgical re-resection, and intraoperative radiation therapy (IORT) as a salvage treatment for patients with recurrent rectal or rectosigmoid carcinoma was recently summarized by Linde $^{30}$. The 5 -year overall survival, local control and di- 
sease-free survival rates of 49 patients receiving IORT were 27,35 , and $20 \%$, respectively. Thirty-four patients who underwent a macroscopic complete resection had a significantly better 5 -year overall survival than the remaining 15 patients with gross residual disease ( 33 vs. $13 \%, P=0.05)$. For those patients, local control and disease-free survival rates were 46 and $27 \%$, respectively. Patients with a microscopic complete resection had a superior 5-year overall survival than partially resected patients (40 vs. $14 \%, P=0.0001$ ). Chemotherapy had no significant influence on overall or disease-free survival. A series treated at Mayo with IORT \pm external irradiation and chemotherapy was recently reported by Haddock ${ }^{25}$. The median, 2-year, and 5-year actuarial overall survivals were 23 months, $48 \%$ and $12 \%$, respectively. The 2 -year actuarial central control was $72 \%$. Local control at 2 years was maintained in $60 \%$ of patients. A prospective study with the aim to investigate the advantage of adding IORT to preoperative irradiation was performed by Wiig ${ }^{60}$ in 107 patients. After surgery, 59 patients had IORT 12-18 Gy. Estimated 5-year survival was overall around $30 \%$. R0-/R1-stage patients survived statistically significantly longer than the R2-group but there was no statistical significant difference between IORT and non-IORT groups in any Rstages regarding overall survival or local recurrence. A recent retrospective study ${ }^{43}$ evaluated 15 patients with pelvic recurrence of colorectal cancer in a previously irradiated region who received IORT (15-20 Gy) as part of salvage therapy. Total prior external radiation therapy doses ranged from 45 to $79 \mathrm{~Gy}$. Tumour resection was accomplished in 14 patients. Three patients received additional postoperative course of $25 \mathrm{~Gy}$. Actuarial 3-year local control and overall survival rates were $25 \%$ and $29 \%$ respectively. Patients with fixed and/or bulky pelvic tumours had a local control rate of $19 \%$ at 12 months. Patients with less extensive clinical presentations had a local control rate of $42 \%$ at 36 months. The authors underlined that clinical presentation of recurrent disease was an important prognostic factor and IORT resulted most advantageous in patients with less extensive clinical presentations.

An interesting study was published by Mannaerts ${ }^{35}$ about the effect on functional outcome after multimodal therapy in 121 patients affected by advanced or recurrent rectal cancer and treated with high-dose preoperative external irradiation, followed by surgery and IORT. The median follow-up was 14 months. A questionnaire distributed to all long-term surviving patients revealed fatigue in $44 \%$, perineal pain in $42 \%$, radiating pain in the leg(s) in $21 \%$, walking difficulties in $36 \%$, and voiding dysfunction in $42 \%$ of the patients as symptoms of ongoing morbidity. Functional impairment consisted of requiring help with basic activities in 15\% and sexual inactivity in $56 \%$ of the respondents. Social handicap was demonstrated by loss of former lifestyle in $44 \%$ and loss of professional occupation in $40 \%$ of patients. This experience underlined the need to weight these consequences against the chance of $\mathrm{Cu}$ re if the patient is treated and the disability eventually caused by uncontrolled tumour progression if the patient is not treated.

\section{Pancreas cancer}

The treatment of pancreatic carcinoma is a challenging situation because of the limited number of operable patients and the high rate of local, regional, and distant relapses. IORT was used in this tumour as part of combined strategy in addiction to surgery, external radiotherapy and in various series combined with systemic chemotherapy. A comprehensive review of the literature data was published by Termehler ${ }^{54}$ who reviewed 21 studies including 727 patients. Doses of IORT varied from $10 \mathrm{~Gy}$ to $40 \mathrm{~Gy}$ usually in addiction to external radiation and chemotherapy obtaining satisfactory pain control in $57-89 \%$ of unresectable patients and median survival of 9-39 months in the patients who underwent tumour resection.

The efficacy of IORT in pancreatic carcinoma seems to be increased by external radiation as shown in a studies conducted at Mayo Clinic $^{18}$ : IORT alone obtained a 2-year local control of $20 \%$ vs. $48 \%$ observed with IORT + external radiation. The Mayo experience demonstrated that IORT can lead to an advantage for local control but not for long-term survival because of the incidence of liver and abdominal metastases. A possible advantage in survival of using preoperative external radiotherapy and IORT rather than postoperative irradiation emerged also from these data. The preoperative external radiotherapy + IORT was tested at MD Anderson by using a short fractionation of 30 Gy in 10 fraction combined with 5 -FU based chemotherapy ${ }^{45}$. Most patients completed the treatment obtaining a 3 -year actuarial survival of $23 \%$ with only $10 \%$ of local failure. An Italian study conducted at San Raffaele Hospital in Milan ${ }^{48}$ showed interesting results in stage I-II disease with a local control of $60 \%$ with IORT and $27 \%$ without IORT and a 6 -year survival of $24 \%$ with IORT and $8 \%$ without IORT.

Recent data from Japan were published by Okamoto ${ }^{41}$ analyzing 132 cases with stage III or IVA pancreatic carcinomas treated by IORT. Sixty-eight patients had locally unresectable tumours and underwent a bypass operation and 64 with resectable tumours underwent pancreatic resection. Postoperative external radiotherapy was also delivered to 90 patients. Multivariate analysis indicated that strong independent predictors of increased survival were external irradiation for unresectable tumours $(p<0.0001)$ and RO in the resected group $(p=0.017)$. Other two recent studies failed to show a significant advantage of using IORT in a combined strategy including external radiotherapy and 5-FU protracted chemotherapy for locally advanced pancreatic carcinoma ${ }^{17}$ and for a series of periampullary cancer treated with radical surgery and conventional postoperative irradiation ${ }^{50}$.

\section{Gastric cancer}

The first demonstration of the feasibility and efficacy of IORT for gastric cancer was reported by $\mathrm{Abe}^{2}$. This experience was followed by other Japanese studies ${ }^{1,53}$ showing that for tumours confined to the gastric wall, IORT was not able to substantially change the prognosis but in case of tumour extension outside the wall and/or lymphnode invasion, IORT significantly increased the local control and the 5-year survival rates. Favourable results were reported by Calvo 6 in 40 patients with stage I-IV disease treated with 10-20 Gy IORT and postoperative irradiation: the 5-year actuarial survival of the entire group was $60 \%$. The relevance of the pathologic lymphnode involvement was pointed out by a study conducted at the University of Lyon ${ }^{22}$ : overall survival ranged from $55 \%$ for $\mathrm{N}+$ to $90 \%$ for $\mathrm{N}$-patients. A recent randomized 


\section{Krengli y cols.}

study comparing IORT combined with preoperative irradiation to surgery alone showed a significant advantage for the IORT arm in the patients with more advanced disease ${ }^{52}$ : the survival for stages PT3-T4 was $60 \%$ with IORT and $15 \%$ without IORT and for $\mathrm{N}+$ was $42 \%$ with IORT and $21 \%$ without IORT.

\section{Biliary tract cancer}

The cancer of the biliary tract is characterized by a high rate of local recurrences. A number of Japanese and US trials using IORT approach were conducted in the attempt to improve the prognosis of this aggressive disease. The experience reported by $\mathrm{Abe}^{1,3}$ did not show any significant improvement of survival rates with IORT compared to external postoperative irradiation. Encouraging results were reported by Todoroki ${ }^{55}$ using preoperative radiotherapy combined with IORT in advanced biliary carcinoma: the 1-year survival rate was $63 \%$. A more recent study of Tsukuba University in Japan $^{56}$ in stage IVA Klatskin tumour showed that adjuvant radiotherapy including IORT yielded significantly higher 5year survival rates $(33.9 \%)$ than in the resection alone group $(13.5 \%)$. Too small was the number of patients accrued in a phase I/II RTOG trial to show any relevant results ${ }^{63}$. A recent Swedish study was reported by Lindell ${ }^{31}$ in 20 patients who underwent extended operation and the last 10 of them IORT and external irradiation in addition. Actuarial 5-year survival was $47 \%$ in the radiotherapy group and $13 \%$ after operation only.

\section{Soft tissue sarcoma}

The necessity to deliver radiotherapy combined with surgery for soft tissue sarcoma is related to the purpose to decrease the occurrence of local relapse. The proximity of radiosensitive critical structures in the retroperitoneal and pelvic regions justifies the use of IORT in combination or not with external irradiation.

The first experience of IORT for the treatment of soft tissue sarcoma was reported by $\mathrm{Abe}^{2}$ with doses of 30-45 Gy and local control in $73 \%$ of patients. A randomized trial was conducted at $\mathrm{NCl}^{51}$ comparing IORT (20 Gy) + external irradiation (35-40 Gy) to external radiotherapy alone (50-55 Gy). Local failure was observed in $20 \%$ of patients treated with IORT and $80 \%$ with external irradiation. Lower incidence of bowel toxicity was found in the IORT arm: $13 \%$ vs. $50 \%$. At $\mathrm{MGH}^{20}, 37$ patients were treated with preoperative irradiation (45-50 Gy) followed by surgery and IORT (15-20 Gy). Local control was obtained in $38 \%$ of patients with 5 -year overall survival of $50 \%$. The best results were observed in the group treated with radical resection with $83 \%$ of local control and $74 \%$ of survival. The Mayo Clinic experience ${ }^{42}$ included 87 patients treated with IORT and external radiotherapy for primary or recurrent disease. The 5-year overall survival was $47 \%$ for the entire group, $52 \%$ for primary and $42 \%$ for recurrent tumours. No significant difference was observed between low- and high-grade sarcomas but a trend was evident in favour of the group with gross total resection in comparison with that with macroscopic residual disease. A French study was performed by Dubois ${ }^{12}$ combining IORT (10-25 Gy) with postoperative external irradiation (45-50 Gy) in completely resected soft tissue sarcoma. Local control was obtained in
$87 \%$ of cases. Other two French studies about retroperitoneal soft tissue sarcoma were published recently. Gilbeau ${ }^{21}$ reported the results obtained 54 patients treated with external postoperative radiotherapy associated with IORT in 17 cases. He observed no difference for local control and survival between the group treated with external radiotherapy alone and external radiotherapy + IORT. Bobin ${ }^{5}$ described a series of 24 patients treated with 15 Gy of IORT and 45-50 Gy of postoperative radiotherapy. Local recurrence were observed in $50 \%$ of cases and disease free survival and overall survival at 5 years were 28 and $56 \%$ respectively.

\section{Gynecologic cancer}

A number of centres have treated metastatic paraaortic nodal disease from primary cervical cancers with external irradiation. Although 15\% to $20 \%$ of such patients appear to be cured, if doses of 55 to 60 Gy are used, complication rates are high. For lower-risk aggressive treatment to this location, lower doses of external radiotherapy could be supplemented by IORT. For patients with relapse in the pelvic side wall or para-aortic nodes, salvage therapy with IORT resulted in overall 5 -years survival of $0 \%$ to $5 \%$ for endometrial cancer and $2 \%$ to $30 \%$ for cervical cancer ${ }^{46}$. In previously irradiated patients, retreatment with meaningful doses of external irradiation is compromised, and use of IORT as a supplement of dose with or without multidrug chemotherapy becomes an option to salvage patients with tumour bed or nodal relapse ${ }^{49}$.

The $\mathrm{Xi}^{\prime}$ an Jiaotong University in China ${ }^{32}$ contributed an analysis of 97 patients with cervical carcinoma treated with preoperative radiotherapy followed by extensive surgery and IORT in the area of high recurrence risk (18-20 Gy) and 85 patients treated with after-loading brachytherapy + external irradiation. The 5-year survival rate in the IORT group was $95 \%$ and that in the single radiotherapy group $88 \%$; for cervical adenocarcinoma, the 5 -year survival rates were $91 \%$ and $33 \%$ respectively. It is concluded that the IORT provided a new therapy method for cervical carcinoma llb especially for cervical adenocarcinoma. There are positive significance for reducing the range of the operation, decreasing complications of radiotherapy, and improving survival quality.

The French Intraoperative Group ${ }^{33}$ presented a study of 70 patients with recurrent uterine cervical carcinoma that underwent IORT alone (40 out of 70) IORT + EBRT (30 out of 70) and additional chemotherapy (20 out of 70). Complete resection (CR) was performed in 30 patients, partial resection (PR) in 37 and unspecified surgery in 3 patients. Median survival and local control rates increased after CR (13 months, $27 \%$ ) vs. PR (15 months, 17\%).

The Clinica Universitaria de Navarra in Spain ${ }^{37}$ presented a study of 31 patients with locally advanced or recurrent cervical cancer treated with simultaneous chemotherapy (CDDP+5-FU) and external irradiation followed by radical surgery with or without IORT boost to the high risk areas for recurrence. The results describe an actuarial disease-free survival of $80 \%$ and a loco-regional control rate of $93.4 \%$. The same institution ${ }^{36}$ performed a retrospective study 26 patients with recurrent gynaecological cancer. This experience comprises two different categories: tumours relapsing after full dose radiation therapy (group I) and recurrent disease to previous surgery (group II). Treatment consisted in maximal 
surgical resection + IORT boost (10-25 Gy) to the high-risk areas for recurrence. Non previously irradiated patients also received external radiotherapy $(+/$ - chemotherapy) pre- or postoperatively. Local control rates have been $33 \%$ and $77 \%$, respectively in groups I and II. The 4-year actuarial overall survival in group I was $7 \%$ and 6 -year actuarial overall survival in group II was $33 \%$. It was concluded that combined external irradiation ( \pm chemotherapy) maximal surgical resection plus IORT could render some long-term survivors among those surgical recurrent patients not candidates for radical surgery with curative intent.

At the Mayo Clinic $^{18}$, a study of 19 patient with recurrent gynaecologic cancer who received IORT and external radiotherapy concluded that IORT in combination with maximum debulking surgery appeared to improve long-term local control and survival.

\section{Bladder cancer}

Most data about the use of IORT in bladder carcinoma come from Japan. $\mathrm{Abe}^{2}$ in a preliminary experience treated $\mathrm{Tl}-$ T4 lesions with 25-30 Gy of IORT combined with 30-40 Gy of postoperative external irradiation obtaining local control in $81-95 \%$ of cases staged T1-T2 and slightly lower rates in more advanced disease. An update of the Japanese experience was performed by Matsumoto ${ }^{38,39}$ on 117 patients. The 5- and 10 -year survival rates were $85 \%$ and $68 \%$ for $\mathrm{Tl}$ and $64 \%$ and $41 \%$ for T2 tumours. In $95 \%$ of patients the bladder function was adequately preserved.

In Spain, Calvo 7,8 conducted a study in 23 patients mostly affected by $\mathrm{T} 3$ lesions combining preoperative external radiotherapy, chemotherapy, cystectomy and IORT with a dose of $15 \mathrm{~Gy}$. No tumour recurrence was detectable in 13 of 17 cases operated with pathologically radical surgery and in all these cases the failure occurred distant. These data suggest that IORT can be very effective when included in a strategy that combines multimodal treatments.

\section{Breast cancer}

Interest in IORT for breast cancer has increased in the last few years thanks to the development of concept of partial breast irradiation. The rationale for the use of this segmental radiation therapy in place of whole-breast irradiation is based on the finding that approximately $85 \%$ of breast relapses are confined to the same quadrant of the breast as the primary tumour. Phase I and II trials have demonstrated no increase in postsurgical complication rates following the use of single-dose IORT in localized breast cancers ${ }^{59}$.

The European Institute of Oncology of Milan in Italy 28,58 presented a study of 185 patients treated with conservative breast surgery + IORT. It was concluded that IORT could allow the course of external radiotherapy to be completely avoided. The authors underlined that IORT dramatically reduced radiation exposure of the skin, lung and subcutaneous tissues and avoided the irradiation of the contralateral breast. It was concluded that single dose IORT affer breast resection for small mammary carcinomas may be an excellent alternative to the traditional postoperative radiotherapy, but a longer follow-up was needed for a better evaluation of the possible late side-effects. Based on the previous results a new study was designed at European Institute of Oncology inclu- ding IORT as solely radiation treatment combined with conservative surgery.

In the study of the Landesklinniken Salzburg Hospital, Austria $^{47}, 156$ patient with stage I and II breast cancer underwent local excision of the fumour and IORT (9 Gy). After wound healing, the patients received additional 51-56 Gy of external irradiation to the whole breast. Also this study suggested that IORT given as a boost after breast conserving surgery could be a reliable alternative to conventional postoperative fractionated boost by accurate dose delivery, avoiding geographical misses, enabling smaller treatment volumes and complete skin-sparing.

\section{Other tumours}

In the literature, a number of preliminary studies regards various tumours that could become elective indications of IORT in the next future.

A recent experience in head and neck and skull base tumours at Mayo Clinic was reported by Pinheiro (44). The study included 34 patients with squamous cell carcinoma (SCC) and 10 patients with non-SCC. Most patients had been previously treated with combinations of surgery, external beam radiotherapy, and chemotherapy. The most frequent sites treated were the skull base $(56 \%)$ and the neck $(44 \%)$. IORT was delivered with energies of 6 to $15 \mathrm{MeV}$ at doses of 12.5 to $22.5 \mathrm{~Gy}$. At 2 years overall and disease-free survival was $32 \%$ and $21 \%$, respectively, for the SCC patients and $50 \%$ and $40 \%$, respectively, for the non-SCC patients. Tumour control rates at 2 years in the IORT field were $46 \%$ for the SCC patients and 52\% for the non-SCC patients. For squamous cell histology, survival in patients with microscopic residual tumour did not differ from those with no residual tumour, but they both had significantly longer disease-free survival than those patients with gross residual at the time of IORT, with a trend toward longer survival.

Also brain tumours have been approached with IORT. The data of a recent Japanese study were reported by Nemoto ${ }^{40}$. Thirty-two cases of high-grade glioma, 11 anaplastic astrocytoma and 21 glioblastoma were surgically resected and received IORT using a dose of 12-15 Gy followed by postoperative external radiation therapy with a dose of $60 \mathrm{~Gy}$. Each of the 32 patients treated with IORT was randomly matched with patients who had been treated with postoperative alone (control). In the anaplastic astrocytoma group, the 1-, 2 - and 5 -year survival rates were $81 \%, 51 \%$ and $15 \%$, respectively in the IORT patients and $54 \%, 43 \%$ and $21 \%$, respectively in the control patients. In the glioblastoma group, 1, 2- and 5 -year survival rates were $63 \%, 26 \%$ and $0 \%$, respectively in the IORT patients and $70 \%, 18 \%$ and $6 \%$, respectively in the control patients. There was no significant difference between survival rates in the IORT patients and control patients in either the anaplastic astrocytoma group or glioblastoma group.

IORT was tested in prostate carcinoma by Kato ${ }^{29}$ in 54 patients staged B2-D1. The treatment included external radiotherapy (30 Gy), IORT (25-30 Gy) through retropubic approach, pelvic lymphadenectomy and hormonal therapy. The 5-year local control and DFS rates were $83 \%$ and $74 \%$ respectively.

An interesting small series of 11 patients with locally recurrent or persistent renal carcinoma following nephrectomy was published by Frydenberg from Mayo $\mathrm{Clinic}^{16}$. The treat- 


\section{Krengli y cols.}

ment included 45-50 Gy preoperative external irradiation followed by maximal surgical debulking and IORT 110-25 Gy.). Of 8 renal cell cancer patients, 6 were alive and 4 were without disease progression at 15 to 50 months ( 3 of 4 at 29 months or longer). One patient died free of disease at 10.5 months and 3 had metastases. Of 3 transitional or SCC patients, 1 died free of disease 28.5 months after initiation of treatment for recurrence and 2 died of disease progression. This experience showed that select patients with solitary local recurrence or persistence following radical nephrectomy for renal cell cancer may benefit from an aggressive local treatment approach using external irradiation + IORT and maximal surgical debulking.

Intra-thoracic tumours such as lung cancer and oesophageal carcinoma were treated with IORT in pilot studies. At University Clinic in Navarra, Madrid Institute of Oncology, and Graduate Hospital in Philadelphia ${ }^{10}$, three different series of stage III non-small-cell lung cancer were treated with IORT after neoadjuvant chemotherapy. The 5-year survival ranged from $20 \%$ to $33 \%$ in the three series and local recurrence rates from $7 \%$ to $40 \%$ for resected and from 50 to $64 \%$ for unresected patients. A Japanese experience of IORT in oesophageal carcinoma was reported by Hosokawa ${ }^{26}$. The patients were treated with mediastinal lymphadenectomy, 12-25 Gy of IORT and 45 Gy of postoperative irradiation. The surgical mortality rate was $0.8 \%$ ( 1 of 121 cases), the overall 5 -year survival rate was $34 \%$ and the cause specific 5 -year survival rate was $55 \%$. The cause specific 5 -year survival rate for $\mathrm{pNO}$ tumours was $79 \%$ and was $44 \%$ for $\mathrm{pN} 1$ tumours. No patients died with locoregional recurrence in the mediastinal lymphnodes.

\section{Conclusions}

In the past, the use of IORT was limited to a very advanced, often recurrent, non-radically operable tumours with poor prognosis. In all these situations, IORT has demonstrated to be feasible with limited toxicity and able to improve the results in most of cases in terms of local control and in a limited number of series also the long-term survival. More recently, IORT has been used in the treatment of early-stage tumours in the attempt to increase local control or improve the quality of life reducing the extension of the radiation treatment volume and consequently the possible long-term side-effects. In this sense, the use of IORT could represent a substantial improvement in a strategy of organ and function preservation of a variety of tumours considering also that the shortening of treatment time contributes to improve the quality of life of the patients.

\section{References}

1. Abe M. History of intraoperative radiation therapy: In: Abe $M$, Dobelbawer RR, eds. Intraoperative Radiation Therapy. Boca Raton: CRC Press 1989; 1.

2. Abe $M$, Takahashi $M$. Intraoperative radiotherapy: The Japanese experience. Int J Radiat Oncol Biol Phys 1981; 7: 863-8.

3. Abe $M$, Takahashi $M$, Yabumoto $E$, et al. Clinical experiences with intraoperative radiotherapy of locally advanced cancers. Cancer 1980; 45:40.
4. Beck C: External roentgen treatment of internal structures eventration treatment. NY Med J 1909; 89:621.

5. Bobin JY, Al-Lawati T, Granero LE, et al. Surgical management of retroperitoneal sarcomas associated with external and intraoperative electron beam radiotherapy. Eur J Surg Oncol 2003; 29:676-81

6. Calvo FA, Henríquez I, Santos $M$, et al. Intraoperative and external beam radiotherapy in locally advanced respectable gastric cancer: Technical description and clinical results. Int J radiat Oncol Biol Phys 1989; 17:183

7. Calvo FA, Aristu JJ, Abuchaibe $O$, et al. Intraoperative and external preoperative radiotherapy in invasive bladder cancer: effect of neoadjuvant chemotherapy in tumor downstaging. Am J Clin Oncol 1993; 16:61-6.

8. Calvo FA, Abuchaibe O, Aristu J. Bladder cancer. In: Calvo FA, Santos M, Brady LW, eds. Intraoperative Radiotherapy: Clinical Experiences and Results. Berlin: Springer-Verlag 1992; 73.

9. Calvo FA, Gómez-Espi M, Díaz-González JA, et al. Intraoperative presacral electron boost following preoperative chemoradiation in T3-4Nx rectal cancer: initial local effects and clinical outcome analysis. Radiother Oncol 2002; 62:201-6.

10. Calvo FA, Meirino RM, Gunderson LL, Willett CG. Intraoperative radiation therapy. In: Perez CA, Brady LW, Halperin EC, Schmidt-Ullrich RK, eds. Principles and Practice of radiation Oncology, Lippincott 2004; 4th Ed 428-56.

11. Canon R, Azinovic I, Aristu JJ, et al. Long-term results after preoperative chemoradiation with or without intraoperative irradiation (IORT) in locally advanced rectal carcinoma. Proc Int Soc Intraoperative Radiation Therapy, Boston 2000; 68.

12. Dubois JB, Debrigode C, Hay M, et al. Intraoperative radiotherapy in soft tissues. Radiother Oncol 1995; 34:160. 13. Eble MJ, Lehnert T, Herfarth $C$, et al: IORT as adjuvant treatment in primary rectal carcinomas: multi-modality treatment. Front $\mathrm{Ra}^{-}$ diat Ther Oncol 1997; 31:200-3.

14. Eloesser L. The treatment of some abdominal cancers by irradiation through the open abdomen combined with cautery excision. Ann Surg 1937; 106:645.

15. Finsterer H. Zur Therapie inoperable Magen und Dormkarzinome mit freilegung und nachfolgender Roentgenbestrahlung. Strahlentherapie 1915; 6:205.

16. Frydenberg M, Gunderson LL, Hahn G, et al. Preoperative external beam radiotherapy followed by cytoreductive surgery and intraoperative radiotherapy for locally advanced primary or recurrent renal malignancies. J Urol 1994; 152:15-21.

17. Furuse J, Kinoshita $T$, Kawashima $M$, et al. Intraoperative and conformal external-beam radiation therapy with protracted 5fluorouracil infusion in patients with locally advanced pancreatic carcinoma. Cancer 2003; 97: 1346-52.

18. Garton GR, Gunderson LL, Nargony DM, et al. High-dose preoperative external beam and intraoperative irradiation for locally advanced pancreatic cancer. Int J radiat Oncol Biol Phys 1993; 27:1153-7.

19. Garton GR, Gunderson LL, Webb MJ, et al. Intraoperative radiation therapy in gynecologic cancer: the Mayo Clinic experience. Gynecol Oncol 1993; 48:328-32.

20. Gieschen HL, Spiro IJ, Suit HD, et al. Long-term results of intraoperative electron beam radiotherapy for primary and recurrent retroperitoneal soft tissue sarcoma. Int J Radiat Oncol Biol Phys 2001 ; 50:127-31.

21. Gilbeau L, Kantor G, Stoeckle E, et al. Surgical resection and radiotherapy for primary retroperitoneal soft tissue sarcoma. Radiother Oncol 2002; 65:137-43. 
22. Glehen $O$, Beaujard $A C$, Romestaing $P$, et al. Intraoperative radiotherapy and external beam radiation therapy in gastric adenocarcinoma with RO-R1 surgical resection. Eur J Surg Oncol 2000; 26 (suppl A):S10-2.

23. Gunderson LL, Cohen AC, Dosoretz DD, et al. Residual unresectable or recurrent colorectal cancer: External beam irradiation and intraoperative electron beam boost +/- resection. Int J Radiat Oncol Biol Phys 1983; 9:1597-606.

24. Gunderson LL, Nagorney DM, Martenson JA, et al: External beam plus intraoperative irradiation for gastrointestinal cancers. World J Surg 1995; 19:191-7.

25. Haddock M, Gunderson L, Nelson H, et al. Intraoperative irradiation for locally recurrent colorectal cancer in previously irradiated patients. Int J Radiat Oncol Biol Phys 2001; 49:126774.

26. Hosokawa $M$, Shirato $H$, Ohara $M$, et al. Intraoperative radiation therapy to the upper mediastinum and nerve-sparing threefield lymphadenectomy followed by external beam radiotherapy for patients with thoracic esophageal carcinoma. Cancer $1999 ; 86: 6-13$

27. Huber FT, Stepan R, Zimmerman F, et al. Locally advanced rectal cancer: resection and intraoperative radiotherapy using the flab method combined with preoperative or postoperative radiochemotherapy. Dis Colon Rectum 1996; 39: 1031-4.

28. Intra M, Gatti $G$, Luini $A$, et al: Surgical techinique of intraoperative radiotherapy in conservative treatment of limited-stage breast cancer. Arch Surg 2002; 137:737-40.

29. Kato $S$, Sakura $M$, Kazumoto $T$, et al: Intraoperative radiation therapy for locally advanced prostate cancer. J Jpn Soc Ther Radiol Oncol 1998; 10:241-8.

30. Lindel K, Willett CG, Shellito PC, et al. Intraoperative radiation therapy for locally advanced recurrent rectal or rectosigmoid cancer. Radiother Oncol 2001; 58:83-7.

31. Lindell G, Holmin T, Ewers $S B$, et al. Extended operation with or without intraoperative (IORT) and external (EBRT) radiotherapy for gallbladder carcinoma. Hepatogastroenterology 2003; 50:310-4

32. Liu Z, Chen X. Preliminary results of intraoperative radiation therapy for cervical carcinoma Ilb. Zhonghua Fu Chan Ke Za Zhi 2002; 37:553-5.

33. Mahe MA, Gerard JP, Dubois JB, et al. Intraoperative radiation therapy in recurrent carcinoma of the uterine cervix: report of the French intraoperative group on 70 patients. Int J Radiat Oncol Biol Phys 1996; 34:21-6.

34. Mannaerts $\mathrm{GH}$, Martijn $\mathrm{H}$, Crommelin MA, et al. Feasibility and first results of multimodality treatment, combining EBRT, extensive surgery, and IOERT in locally advanced primary rectal cancer. Int J Radiat Oncol Biol Phys 2000; 47:425-33.

35. Mannaerts $\mathrm{GH}$, Rutten $\mathrm{HJ}$, Martijn $\mathrm{H}$, et al: Effects on functional outcome after IORT-containing multimodality treatment for locally advanced primary and locally recurrent rectal cancer. Int J Radiat Oncol Biol Phys 2002; 54:1082-8.

36. Martínez MR, Jurado $M$, Azinovic l, et al. Intraoperative radiotherapy in recurrent gynecologic cancer. Radiother Oncol 1993, 28:127-33

37. Martínez MR, Jurado M, Azinovic I, et al. Preoperative chemoradiation and adjuvant surgery in locally advanced or recurrent cervical carcinoma. Rev Med Univ Navarra 1997; 41:19-26.

38. Matsumoto L, Kakizoe T, Mikuriya S, et al. Clinical evaluation of intraoperative radiotherapy for carcinoma of the urinary bladder. Cancer 1981; 47:509.
39. Matsumoto L. Intraoperative radiation therapy for bladder cancer. In: Dobelbower RR, Abe M, eds. Intraoperative Radiation Therapy. Boca Raton: CRC Press 1989; 217.

40. Nemoto K, Ogawa $Y$, Matsushita $\mathrm{H}$, et al: Intraoperative radiation therapy (IORT) for previously untreated malignant gliomas. BMC Cancer 2002; 2:1.

41. Okamoto A, Tsuruta K, Karasawa K, et al. Resection versus paIliation: treatment of stage III and IVA carcinomas of the pancreas employing intraoperative radiation. World J Surg 2003; 27:599-605.

42. Petersen IA, Haddok MG, Denohne JH, et al. Use of intraoperative electron beam radiotherapy in the management of retroperitoneal soft tissue sarcomas. Int J Radiat Oncol Biol Phys 2002; 52:469-75

43. Pezner RD, Chu DZ, Ellenhorn JD. Intraoperative radiation therapy for patients with recurrent rectal and sigmoid colon cancer in previously irradiated fields. Radiother Oncol 2002; 64:47-52.

44. Pinheiro AD, Foote RL, McCaffrey TV, et al: Intraoperative radiotherapy for head and neck and skull base cancer. Head Neck 2003; 25:217-25.

45. Pisters PW, Abruzzese JL, Janjan NA, et al. Rapid-fractionation preoperative chemoradiation, pancreaticoduodenectomy, and intraoperative radiation therapy for respectable pancreatic adenocarcinoma. J Clin Oncol 1998; 16:3843-50.

46. Piver MS, Barlow JJ. High dose irradiation in biopsy confirmed aortic node metastases from carcinoma of the uterine cervix. Cancer 1977; 39:1243-6.

47. Reitsamer R, Peintinger F, Sedlmayer F, et al. Intraoperative radiotherapy given as a boost after breast-conserving surgery in breast cancer patients. Eur J Cancer 2002; 38:1607-10.

48. Reni $M$, Panucci MG, Ferreri $A J$, et al. Effect on local control and survival of electron beam intraoperative irradiation for respectable pancreatic adenocarcinoma. Int J Radiat Oncol Biol Phys 2001; 50:651-8.

49. Rich TA. Intraoperative Radiation Therapy. In: Perez CA, Brady LW, Principles and Practice of Radiation Oncology, Third Ed., Lippincott-Raven 1997; 629-35.

50. Schwarz RE, Smith DD, Keny H, et al. Impact of intraoperative radiation on postoperative and disease-specific outcome after pancreatoduodenectomy for adenocarcinoma: a propensity score analysis. Am J Clin Oncol 2003; 26:16-21.

51. Sindelar WF, Kinsella TJ, Chen PW, et al. Intraoperative radiotherapy in retroperitoneal sarcomas: Final results of a prospective, randomized trial. Arch Surg 1993; 128:402-10.

52. Skoropad VY, Berdov BA, Mardynski YS, et al. A prospective, randomized trial of pre-operative and intraoperative radiotherapy versus surgery alone in resectable gastric cancer. Eur J Surg Oncol 2000; 26:773-9.

53. Takahashi $M$, Abe $M$. Intraoperative radiation therapy for carcinoma of the stomach. Eur J Surg Oncol 1986; 12: 247.

54. Termehler PM, Evans DB, Willet CG, et al. IORT in pancreatic cancer. In: Gunderson LL, Willett CG, Harrison LB, et al, eds. Intraoperative irradiation. Techniques and results. Totowa, New Jersey: Humana Press 1999; 201-2.

55. Todoroki T, Iwasaki Y, Okamura T, et al. Intraoperative radiotherapy for advanced carcinoma of the biliary system. Cancer $1980 ; 46: 2179$.

56. Todoroki T, Ohara K, Kawamoto T, et al. Benefits of adjuvant radiotherapy after radical resection of locally advanced main hepatic duct carcinoma. Int J radiat Oncol Biol Phys 2000; 46:581-7. 


\section{Krengli y cols.}

57. Valentini V, Balducci $M$, Trodella $L$, et al. Preoperative radiotherapy plus IORT in T3 rectal cancer. Proc Int Soc Intraoperative Radiation Therapy, Boston 2000:68.

58. Veronesi $U$, Orecchia $R$, Luini $A$, et al: A preliminary report of intraoperative radiotherapy (IORT) in limited-stage breast cancers that are conservatively treated. Eur J Cancer 2001 37:2178-83.

59. Veronesi $U$, Gatti $G$, Luini $A$ et al. Intraoperative radiation therapy for breast cancer: technical notes. Brest J 2003; 9: 10612.

60. Wiig JN, Tveit KM, Poulsen JP, et al. Preoperative irradiation and surgery for recurrent rectal cancer. Will intraoperative ra- diotherapy (IORT) be of additional benefit? A prospective study. Radiother Oncol 2002; 62:207-13.

61. Willett CG, Shellito PC, Tepper JE, et al. Intraoperative electron beam radiation therapy for primery locally advanced rectal and rectosigmoid carcinoma. J Clin Oncol 1991; 9: 843-9.

62. Willett CG, Shellito PC, Gunderson LL: Primary colorectal EBRT and IOERT. In: Gunderson LL, Willett CG, Harrison LB, et al, eds. Intraoperative irradiation techniques and results. Totowa, New Jersey: Humana Press, 249-272, 1999.

63. Wolkov HB, Graves $G$, Wen $M$, et al: Intraoperative radiation therapy of extrahepatic biliary carcinoma: A report of RTOG 8506. Am J Clin Oncol, 15: 323, 1992. 